\title{
Advanced parental age in maternal uniparental disomy (UPD): implications for the mechanism of formation
}

\author{
Dieter Kotzot*,1 \\ ${ }^{1}$ Institute of Medical Biology and Human Genetics, University of Innsbruck, Innsbruck, Austria
}

Uniparental disomy (UPD) describes the inheritance of a pair of chromosomes from only one parent. Meiotic nondisjunction followed by trisomy rescue is considered to be the major mechanism of formation. A literature search for cases with whole chromosome UPD other than UPD 15 was performed. Information on parental age was available in 111 cases with maternal UPD and in 34 cases with paternal UPD. In 52 out of 74 cases with maternal heterodisomy, information on the time of nondisjunction was also available. Around two-thirds of these cases were due to a maternal meiosis I error. Compared with the mean maternal age of 30.0 years in Bavarian mothers, in the year 2000 an advanced mean maternal age of 34.8 years was found in cases with maternal heterodisomy $(n=74 ; P<0.0001)$. Almost no difference in the mean maternal age was observed between meiosis I errors (35.56 years; $n=30)$ and meiosis II errors $(35.78$ years; $n=14)$. The mean maternal age was 31.46 years in cases with maternal isodisomy and a normal karyotype $(n=24)$, and the mean paternal age was 31.48 years in cases with paternal isodisomy $(n=28)$. The various mean parental ages in heterodisomic and isodisomic cases are considered to reflect strongly the different mechanisms of formation: trisomy rescue or gamete complementation, which implies a meiotic nondisjunction in maternal heterodisomic UPD, and postzygotic somatic reduplication in cases with paternal and maternal isodisomic UPD.

European Journal of Human Genetics (2004) 12, 343-346. doi:10.1038/sj.ejhg.5201158

Published online 28 January 2004

Keywords: heterodisomy; isodisomy; parental age; uniparental disomy

\section{Introduction}

Uniparental disomy (UPD) describes the inheritance of a pair of chromosomes from only one parent. Inheritance of both or parts of both maternal homologs (heterodisomic UPD) has been described in the majority of cases (reviewed in $\operatorname{Kotzot}^{1}$ ). Mechanisms of formation discussed in the literature are trisomy rescue, monosomy rescue (or somatic reduplication), gamete complementation, and postfertilization error. ${ }^{2}$ In each of these mechanisms, two events are

*Correspondence: Dr med. D Kotzot, Institute of Medical Biology and Human Genetics, Schoepfstr. 41, A-6020 Innsbruck, Austria.

Tel: + 43512507 3464; Fax: + 43512507 2861;

E-mail: DieterKotzot@gmx.de

Received 26 September 2003; revised 22 November 2003; accepted 3 December 2003 necessary, either two meiotic, or two mitotic, or one meiotic and one mitotic event. Most of them are chromosomal nondisjunction events, which in general are also the major mechanism of formation of classical aneuploidy, and their frequency correlate to the mean maternal age (ie, trisomy 21). ${ }^{3-5}$ Already in 1993 Robinson et $a l^{6}$ reported on increased mean parental ages in cases with UPD 15. Using a similar approach in 1996, the same author analyzed the age-specific risks for UPD(15) and found an exponential increase with maternal age similar to that observed for trisomy 21. ${ }^{7}$ Ginsburg et $a l^{8}$ found an increase of maternal UPD 15, paternal UPD 15, and maternal UPD 7 in the offspring of mothers older than 35 years at delivery. One can therefore hypothesize that in general, there is also a correlation between the mean 
parental age and UPD, and that in particular, the mean maternal age is expected to increase in cases with maternal heterodisomic UPD.

Here, by searching all cases with UPD other than UPD 15 so far reported in the literature, a preponderance of maternal meiosis I errors and a strongly advanced mean maternal age of 34.9 years was found in cases with a normal karyotype and maternal heterodisomy compared to the mean maternal age of 30.0 years in the general population.

\section{Methods and results}

An extensive Medline search for cases with either complete maternal or complete paternal UPD other than UPD 15 published until October of 2003 was performed. In total, 181 cases with maternal UPD (106 X heterodisomy, $53 \mathrm{X}$ isodisomy, and 22 without further specification) and 63 cases with paternal UPD (12 X heterodisomy, $45 \mathrm{X}$ isodisomy, and six without further specification) were found (data not reported in detail). Each of these cases was screened for information on parental age. In cases where the original publication did not contain this information, the corresponding author was contacted. Eventually, parental age was available for 111 cases with maternal UPD (approx. 61\%) and 34 cases with paternal UPD (approx. 54\%) (Table 1). Data on molecular investigations were evaluated separately for maternal and paternal UPD and within each of these groups for cases with a $46, \mathrm{XX}$ or a $46, \mathrm{XY}$ karyotype and for cases with any chromosome aberration (Table 2). Exact references for each case included in the study are listed in the Appendix. Heterozygosity for proximal loci and homozygosity for distal loci were considered as a consequence of a meiosis I error, whereas the opposite should indicate a meiosis II nondisjunction. Due to insufficient information in some heterodisomic cases, there were difficulties in defining the time of nondisjunction. For statistical analysis the one sample $t$-test was applied (http://www.graphpad.com/ quickcalcs/index.cfm), but the result should be interpreted carefully as the data presented here include cases from northern European countries with a high mean maternal age and from countries with a very low mean maternal age.

In cases with maternal heterodisomy and a normal karyotype, a preponderance of maternal meiosis I errors was observed (30 out of 44 ). The mean maternal age was significantly increased in cases with a UPD due to maternal meiotic nondisjunction (34.8 years, $P<0.0001)$ compared with the mean maternal age of Bavarian mothers (30.0 years in the year 2000), which comprise a population of approx. 6.3 million women and approx. 120000 births a year (Bayerisches Landesamt für Statistik und Datenverarbeitung, München). Statistical significance was found in all groups of maternal heterodisomy (Table 2). In contrast, the mean maternal age was in the normal range in cases
Table 1 Number of cases with UPD evaluated for parental age (total number of cases reported in the literature)

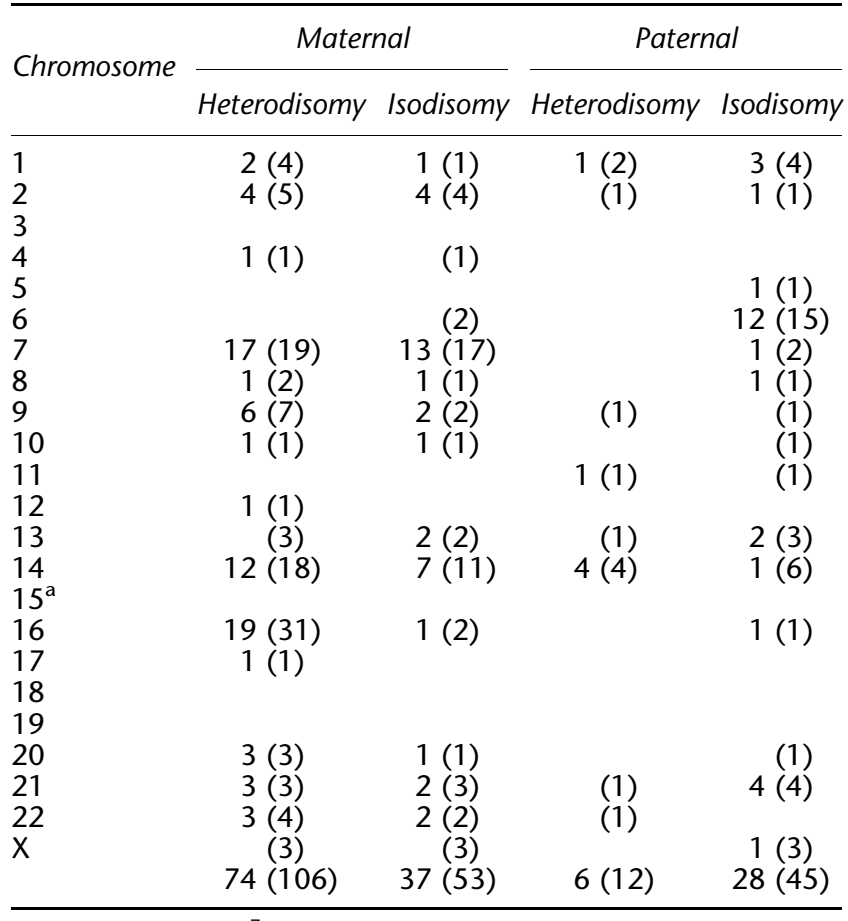

${ }^{a}$ See Robinson et al. ${ }^{7}$

with isodisomy (29.87 years) (Table 2) The difference between the mean maternal age of all cases with maternal heterodisomy (34.8 years, $n=74$ years) and the mean maternal age of cases with maternal isodisomy $(29.87$ years, $n=37)(P<0.0005)$ is statistically significant when compared using a simple $t$-test (http://www.graphpad.com/ quickcalcs/index.cfm). In the subgroup of maternal heterodisomic UPD of acrocentric chromosomes (with and without chromosome aberration, $n=17$ ), the mean maternal age was 34.47 years, which is not significantly different from the total mean maternal age of cases with heterodisomic maternal UPD. Owing to the few cases, delineation of meiosis I errors $(n=7)$ versus meiosis II errors $(n=0)$ versus unknown time of meiotic nondisjunction $(n=3)$ versus cases with a chromosome aberration $(n=7)$ was not possible. The separate analysis of UPD of single chromosomes revealed mean maternal ages of 36.1 years for maternal heterodisomic UPD $7(n=17)$ and 33.7 years for maternal heterodisomic UPD $16(n=19)$, as well as a mean paternal age of 29.3 years for cases with paternal isodisomic UPD $6(n=12)$. All these results are not significantly different from the mean parental age of the corresponding group.

\section{Discussion}

Advanced maternal age is a major risk factor for chromosome nondisjunction. Almost 70 years ago, Penrose 
Table 2 Mean parental age for various forms of UPD

\begin{tabular}{|c|c|c|}
\hline & Mean paternal age $(n)$ & Mean maternal age ( $n)$ \\
\hline \multicolumn{3}{|l|}{ Maternal heterodisomy } \\
\hline Meiosis I & $36.66(12)$ & $35.56(30)(P<0.0001)$ \\
\hline Meiosis II & $37.4(10)$ & $35.78(14)(P<0.01)$ \\
\hline Meiosis ? & $36.2(5)$ & $33.32(19)(P<0.0001)$ \\
\hline Meiosis I+Meiosis II+Meiosis ? & $36.85(27)$ & $34.9(63)(P<0.0001)$ \\
\hline Meiosis I+chromosome aberration & $35.5(4)$ & $34.5(6)(P<0.07)$ \\
\hline Meiosis II+chromosome aberration & $38.5(2)$ & $36.5(2)$ \\
\hline Meiosis ?+chromosome aberration & $36.6(3)$ & $31.66(3)$ \\
\hline Total & $34.0(36)$ & $34.8(74)(P<0.0001)$ \\
\hline \multicolumn{3}{|l|}{ Maternal isodisomy } \\
\hline $46, \mathrm{XX}$ or $46, \mathrm{XY}$ & $30.56(16)$ & $31.46(24)(\mathrm{NS})$ \\
\hline Chromosome aberration & $27.63(8)$ & $26.92(13)(\mathrm{NS})$ \\
\hline Total & $29.58(24)$ & $29.87(37)(\mathrm{NS})$ \\
\hline \multicolumn{3}{|l|}{ Paternal heterodisomy } \\
\hline Meiosis I+II & $32.5(4)$ & $32.0(4)$ \\
\hline Chromosome aberration & $25.5(2)$ & $24.5(2)$ \\
\hline Total & $30.2(6)$ & $27.83(6)$ \\
\hline \multicolumn{3}{|l|}{ Paternal isodisomy } \\
\hline $46, X X$ or $46, X Y$ & $30.78(23)$ & $27.92(25)$ \\
\hline Chromosome aberration & $34.4(5)$ & $29.5(8)$ \\
\hline Total & $31.42(28)$ & $28.3(33)$ \\
\hline
\end{tabular}

The various mean maternal ages were compared with the mean maternal age of 30 years of Bavarian mothers in the year 2000 .

recognized a correlation between maternal age and the risk to deliver a baby with Down syndrome. ${ }^{9}$ About 60 years later, the correlation with mean maternal age was proven. In addition, it was demonstrated that approx. $68 \%$ of cases with maternally derived trisomy 21 can be attributed to a nondisjunction event in maternal meiosis I (reviewed in Antonarakis ${ }^{5}$ ) as due to the small number of cases, data for a paternal age effect in trisomy 21 and other numerical chromosome aberrations were ambiguous (reviewed in Gardner and Sutherland ${ }^{10}$ ).

In UPD, the situation is even more difficult as the total number of cases is low. So far, only 181 cases with maternal UPD and 63 cases with paternal UPD other than UPD 15 have been reported (Table 1 and Appendix). Information on parental age and the time of nondisjunction was available for totally only 91 cases with maternal UPD and 34 cases with paternal UPD (Table 1 ). In contrast to trisomy $13,18,21$, and $\mathrm{X}$ and monosomy $\mathrm{X}$, almost all fetuses with nonmosaic and even mosaic aneuploidy of other chromosomes are spontaneously aborted early in pregnancy. Therefore, only initially aneuploid zygotes in which additionally formation of UPD had occurred very early in embryonic development might come to our attention. The later any correction occurs, the more likely the pregnancy will diminish and UPD goes undetected. Moreover, in trisomy rescue by theory two-thirds of all cases should have biparental disomy and would therefore not have been recognized. For some chromosomes, no case of UPD has been described so far. Apart from the phenotypical effects of UPD due to genomic imprinting in paternal UPD 6, maternal UPD 7, paternal UPD 11p15, maternal UPD 14, paternal UPD 14, maternal UPD 15, and paternal UPD 15 clinical consequences of UPD have not been proven so far or will only be ascertained by homozygosity of an autosomal recessively inherited mutation due to isodisomy. Therefore, one can assume that the majority of cases with UPD go undetected. In addition, all systematic studies published so far focused on a specific clinical phenotype (reviewed in $\operatorname{Kotzot}^{11}$ ). The data presented here comprise cases from all over the world and parental age at birth is also strongly influenced by cultural and socio-economic effects. In populations with a high mean maternal age, the frequency of UPD should be higher and the mean maternal age in UPD cases should also be higher. Vice versa, the effect of mean maternal age on the formation of trisomy, and therefore the frequency of UPD, should be more pronounced in populations with a low mean maternal age. Cases of UPD associated with a Robertsonian translocation or any other chromosome aberration might be a consequence not only of a simple nondisjunction event but also of a more complex mechanism of formation.

Keeping all these factors in mind, nevertheless, the majority of maternal UPD cases is associated with a meiosis I nondisjunction and there is a trend of an advanced mean maternal age in cases with maternal heterodisomy (34.8 years) compared to children with biparental inheritance (30.0 years in Bavaria in the year of 2000). Both the increased mean maternal age and the preponderance of meiosis I errors are in good agreement with the findings in the specific situation of maternal and paternal UPD $15^{6-8}$ 
and in classical trisomy, ${ }^{3,4}$ and therefore, are a strong hint for the suspected formation due to gamete complementation or trisomy rescue. Support for the latter also comes from cases with proven low-level mosaicism. The similarly increased mean paternal age (34.0 years, $n=36)$ in cases with maternal heterodisomy might be due to either sociocultural reasons or being a hint towards simultaneous aneuploidy in sperms resulting in gamete complementation. However, in cases without detectable trisomy mosaicism, it is not possible to delineate UPD due to trisomy rescue from UPD due to gamete complementation.

The normal mean paternal age in paternal isodisomy might also be considered as a hint for somatic reduplication subsequent to the fertilization of a hypohaploid egg by a normal sperm as the major mechanism of formation in this group. As the proportion of hypo- or hyperhaploidy is lower in sperms than in eggs (3-4 versus $18-19 \%)$, both in general and in correlation with the paternal age, ${ }^{12}$ it is less likely that a hypohaploid egg is fertilized by a hyperhaploid sperm that would result in gamete complementation than by a normal sperm followed by somatic reduplication. Otherwise, the high number of isodisomic cases can only be explained by hyperhaploid sperms associated with a lack of recombination in paternal meiosis producing sperms with two copies of one homolog. Interpretation of the mean paternal age in cases with paternal heterodisomy was not possible due to the very low number of only six cases.

In summary, there is a strongly increased mean maternal age in cases with maternal heterodisomic UPD compared to cases with isodisomy and to the general population. These findings are a further argument to consider as the major mechanism of formation: trisomy rescue and gamete complementation in maternal heterodisomic UPD and somatic reduplication in paternal isodisomic UPD.

\section{References}

1 Kotzot D: Abnormal phenotypes in uniparental disomy (UPD) fundamental aspects and a critical review with bibliography of UPD other than 15. Am J Med Genet 1999; 82: 265-274.

2 Robinson WP: Mechanisms leading to uniparental disomy and their clinical consequences. BioEssays 2000; 22: 452-459.

3 Hassold TJ, Jacobs PA: Trisomy in man. Annu Rev Genet 1984; 18: 69-97.

4 Hassold T, Hunt P: To err (meiotically) is human: the genesis of human aneuploidy. Nat Rev Genet 2001; 2: 280-291.

5 Antonarakis SE: 10 years of Genomics, chromosome 21, and Down syndrome. Genomics 1998; 51: 1-16.

6 Robinson WP, Lorda-Sanchez I, Malcolm S et al: Increased parental ages and uniparental disomy 15: a paternal age effect? Eur J Hum Genet 1993; 1: 280-286.

7 Robinson WP, Langlois S, Schuffenhauer S et al: Cytogenetic and age-dependent risk factors associated with uniparental disomy 15. Prenatal Diagn 1996; 16: 837-844.

8 Ginsburg C, Fokstuen S, Schinzel A: Contribution of uniparental disomy to congenital development defects in children born to mothers at advanced childbearing age. Am J Med Genet 2000; 95: 454-460.

9 Penrose L: The relative effects of paternal and maternal age in mongolism. J Genet 1933; 27: 219-224.

10 Gardner RJM, Sutherland GR: Chromosome Abnormalities and Genetic Counselling. Oxford: Oxford University Press; 1996.

11 Kotzot D: Review and meta-analysis of systematic searches for uniparental disomy (UPD) other than UPD 15. Am J Med Genet 2002; 111: 366-375.

12 Martin RH, Ko E, Rademaker A: Distribution of aneuploidy in human gametes: comparison between human sperm and oocytes. Am J Med Genet 1991; 39: 321-331.

(Supplementary Information accompanies the paper on European Journal of Human Genetics website (http://www. nature.com/ejhg)). 